\title{
A Simple Proof of the GHS and Further Inequalities
}

\author{
Richard S. Ellis` \\ Department of Mathematics, Northwestern University, Evanston, Illinois, USA \\ James L. Monroe ${ }^{\star}$ \\ Department of Physics, Northwestern University, Evanston, Illinois, USA
}

Received October 16, 1974

\begin{abstract}
We formulate and prove a general set of correlation inequalities for spin - 1/2 Ising ferromagnets with pair interactions. One of these is the Griffiths-Hurst-Sherman inequality. The proof is obtained using Gaussian random variables.
\end{abstract}

\section{Introduction}

We consider a system of $N$ Ising spins with ferromagnetic pair interactions and non-negative external magnetic field. The probability $\mu(\sigma)$ of any configuration $\sigma=\left(\sigma_{1}, \ldots, \sigma_{N}\right), \sigma_{i}= \pm 1$, is given by the formula $\mu(\sigma)=Z^{-1} \exp (-\beta H(\sigma))$, where $\beta=(k T)^{-1}$,

$$
\begin{aligned}
H(\sigma) & =-\frac{1}{2} \sum_{i \neq j} J_{i j} \sigma_{i} \sigma_{j}-h \sum_{i} \sigma_{i}, \quad J_{i j}=J_{j i} \geqq 0, \quad h \geqq 0, \\
Z & =\sum_{\{\sigma\}} \exp (-\beta H(\sigma)) .
\end{aligned}
$$

In the sequel, we set $\beta=1$. Given spin sites $i, j, k$, we define the third Ursell function

$$
\begin{aligned}
u_{3}(i, j, k) \equiv & \left\langle\sigma_{i} \sigma_{j} \sigma_{k}\right\rangle-\left\langle\sigma_{i}\right\rangle\left\langle\sigma_{j} \sigma_{k}\right\rangle-\left\langle\sigma_{j}\right\rangle\left\langle\sigma_{i} \sigma_{k}\right\rangle \\
& -\left\langle\sigma_{k}\right\rangle\left\langle\sigma_{i} \sigma_{j}\right\rangle+2\left\langle\sigma_{i}\right\rangle\left\langle\sigma_{j}\right\rangle\left\langle\sigma_{k}\right\rangle,
\end{aligned}
$$

where the bracket \langle\rangle denotes the expected value with respect to the measure $\mu$.

The Griffiths-Hurst-Sherman inequality (hereafter GHS inequality) states that

$$
u_{3}(i, j, k) \leqq 0 .
$$

An important consequence of this inequality is that the average magnetization per site is a concave function of magnetic field $h$, a fact needed for the proof of certain critical point exponent inequalities [1]. It has also been used by Preston [2] to show the absence of phase transitions in the thermodynamic limit for $h \neq 0$.

Inequality (1.4) was first proved by Griffiths, Hurst, and Sherman [1] and later by Lebowitz [3]. Our proof is completely self-contained and, we believe, is much simpler. It is based on ideas introduced by Monroe and Siegert [4], who obtained simple proofs of the GKS inequalities [5]. Similar methods have also been used by Monroe [6] to prove certain FKG inequalities [7]. At the end of the next section, we mention additional new inequalities which are proved by the same technique.

* Supported in part by National Science Foundation Grant GP-28576.

$\star \star$ Supported by National Science Foundation Grant GP-36564-XI. 


\section{Sketch of Proof and Further Results}

For convenience, we assume $i=1, j=2, k=3$. Our proof is based on the identity [8]

$$
\begin{aligned}
& \exp \left[\frac{1}{2} \sum_{i, j} \xi_{i} v_{i j} \xi_{j}\right] \\
& \quad=(2 \pi)^{-N / 2}(\operatorname{det} v)^{-1 / 2} \int \ldots \int \prod_{i=1}^{N} d x_{i} \exp \left[-\frac{1}{2} \sum_{i, j} x_{i}\left(v^{-1}\right)_{i j} x_{j}+\sum_{i} \xi_{i} x_{i}\right],
\end{aligned}
$$

valid for any symmetric, real, positive definite matrix $v$ and for any $N$ complex variables $\xi_{i}$. The right-hand side of (2.1) can be considered as the expected value $E_{x}\left[\exp \Sigma_{1 \leqq i \leqq N} x_{i} \xi_{i}\right]$ with respect to the Gaussian density function

$$
f_{v}(\bar{x})=(2 \pi)^{-N / 2}(\operatorname{det} v)^{-1 / 2} \exp \left[-\frac{1}{2} \sum_{i, j} x_{i}\left(v^{-1}\right)_{i j} x_{j}\right]
$$

where $\bar{x}=\left(x_{1}, \ldots, x_{N}\right)$. If $v$ is a non-negative matrix, then given integers $n_{i} \geqq 0$, one can show

$$
E_{x}\left[\prod_{i=1}^{N}\left(x_{i}\right)^{n_{i}}\right]\left\{\begin{array}{lll}
=0 & \text { if } & \sum_{i=1}^{N} n_{i} \text { is odd }, \\
\geqq 0 & \text { if } \sum_{i=1}^{N} n_{i} \text { is even . }
\end{array}\right.
$$

To use (2.1), we identify the variables $\xi_{i}$ with the spin variables $\sigma_{i}$ and form a matrix $v=J$ with off-diagonal elements $J_{i j}$, diagonal elements all equal to a number $J_{0} \equiv J_{i i}$ large enough to guarantee that $J$ is positive definite. We then let $\left(x_{1}, \ldots, x_{N}\right),\left(y_{1}, \ldots, y_{N}\right),\left(z_{1}, \ldots, z_{N}\right),\left(w_{1}, \ldots, w_{N}\right)$ be independent sets of Gaussian random variables, where the random variables in each set have joint density function $f_{J}$. Writing $\bar{E}$ to denote expectation with respect to the product measure $f_{J}(\bar{z}) f_{J}(\bar{y}) f_{J}(\bar{z}) f_{J}(\bar{w})$, we show that

$$
u_{3}(1,2,3)=K \cdot \bar{E}\left[D\left(\frac{\partial}{\partial x_{l}}, \frac{\partial}{\partial y_{l}}, \frac{\partial}{\partial z_{l}}\right) g(\bar{x}, \bar{y}, \bar{z}, \bar{w})\right]
$$

where $K$ is some positive constant, $D$ denotes a sum of products of partial derivatives involving $\frac{\partial}{\partial x_{l}}, \frac{\partial}{\partial y_{l}}, \frac{\partial}{\partial z_{l}}, l=1,2,3$, and $g$ is a certain function of $\bar{x}, \bar{y}, \bar{z}, \bar{w}$.

The crux of the proof is to reexpress (2.4) in terms of new variables $\bar{\alpha}=\left\{\alpha_{l}\right\}, \bar{\beta}=\left\{\beta_{l}\right\}$, $\bar{\gamma}=\left\{\gamma_{l}\right\}$, and $\bar{\delta}=\left\{\delta_{l}\right\}, l=1, \ldots, N$, obtained by a certain orthogonal transformation of $\bar{x}, \bar{y}, \bar{z}, \bar{w}$. A remarkable simplification then occurs, and we have

$$
u_{3}(1,2,3)=2 K \cdot \bar{E}\left[\frac{\partial}{\partial \beta_{1}} \frac{\partial}{\partial \gamma_{2}} \frac{\partial}{\partial \delta_{3}} \tilde{g}(\alpha, \beta, \gamma, \delta)\right],
$$

where $\tilde{g}$ is the transformed $g$. The GHS inequality (1.4) then follows from (2.3).

Our method has the following consequence. Let $\tilde{\mathscr{D}}$ denote a product of partial derivatives of the form

$$
\prod_{i, j, k, l}\left(\frac{\partial}{\partial \alpha_{i}}\right)^{n_{i} \alpha}\left(\frac{\partial}{\partial \beta_{j}}\right)^{n_{j} \beta}\left(\frac{\partial}{\partial \gamma_{k}}\right)^{n_{k} \gamma}\left(\frac{\partial}{\partial \delta_{l}}\right)^{n_{l} \delta}
$$


where $i, j, k, l$ range over all lattice points $\{1, \ldots, N\}$ and $n_{i}^{\alpha}, n_{j}{ }^{\beta}, n_{k}{ }^{\gamma}, n_{l}{ }^{\delta}$ are nonnegative integers. Each such $\tilde{\mathscr{D}}$ gives rise to a sum $c_{\mathscr{D}}$ of correlations.

Theorem 1. Define

$$
N_{1}=\sum_{i=1}^{N} n_{i}^{\alpha}, \quad N_{2}=\sum_{j=1}^{N} n_{j}^{\beta}, \quad N_{3}=\sum_{k=1}^{N} n_{k}^{\gamma}, \quad \text { and } \quad N_{4}=\sum_{l=1}^{N} n_{l}^{\delta} .
$$

We then have the following:

a) $c_{\mathscr{D}} \leqq 0$, if each $N_{i}, i=1, \ldots, 4$, is odd;

b) $c_{\mathscr{D}} \geqq 0$, if each $N_{i}, i=1, \ldots, 4$, is even:

c) $c_{\mathscr{D}}=0$, in all other cases.

When $h>0$, the oddness or evenness of $N_{1}$ is not to be considered.

The simplest of these $\tilde{\mathscr{D}}$ correspond to elementary GKS inequalities; e.g., $\tilde{\mathscr{D}}=\partial / \partial \alpha_{i}$ gives rise to $\left\langle\sigma_{i}\right\rangle \geqq 0 ; \tilde{\mathscr{D}}=\left(\partial / \partial \beta_{i}\right)\left(\partial / \partial \beta_{j}\right)$ gives rise to $\left\langle\sigma_{i} \sigma_{j}\right\rangle$ $-\left\langle\sigma_{i}\right\rangle\left\langle\sigma_{j}\right\rangle \geqq 0$ (see Appendix for calculations). In future work, we shall investigate the explicit forms, in terms of the spin variables $\sigma_{i}$, of the inequalities in Theorem 1 as well as generalizations of them.

\section{Proof of GHS Inequality and Theorem 1}

We first note that the Bolzmann factor $\exp (-H(\sigma))$ can be written as

$$
\begin{aligned}
\exp (-H(\sigma)) & =E_{\bar{x}}\left[\exp \left\{\sum_{i=1}^{N}\left(\left[x_{i}+h\right] \sigma_{i}-\frac{1}{2} J_{0} \sigma_{i}^{2}\right)\right\}\right] \\
& =\exp \left(-\frac{N J_{0}}{2}\right) E_{\bar{x}}\left[\prod_{i=1}^{N} \exp \left\{\left(x_{i}+h\right) \sigma_{i}\right\}\right],
\end{aligned}
$$

since each $\sigma_{i}^{2}=1$. Thus,

and, for example

$$
Z=2^{N} \exp \left(-\frac{N J_{0}}{2}\right) E_{\bar{x}}\left[\prod_{i=1}^{N} \cosh \left(x_{i}+h\right)\right],
$$

$$
\begin{aligned}
\left\langle\sigma_{1}\right\rangle\left\langle\sigma_{2} \sigma_{3}\right\rangle= & {\left[\frac{1}{Z} \sum_{\{\sigma\}} \sigma_{1} \exp (-H(\sigma))\right]\left[\frac{1}{Z} \sum_{\left\{\sigma^{\prime}\right\}} \sigma_{2}^{\prime} \sigma_{3}^{\prime} \exp \left(-H\left(\sigma^{\prime}\right)\right)\right] } \\
= & 2^{2 N} Z^{-2} \exp \left(-N J_{0}\right)\left\{\sum_{\{\sigma\}} E_{\bar{x}}\left[\frac{\partial}{\partial x_{1}} \prod_{i=1}^{N} \exp \left[\left(x_{i}+h\right) \sigma_{i}\right]\right] .\right. \\
& \left.\sum_{\left\{\sigma^{\prime}\right\}} E_{\bar{y}}\left[\frac{\partial}{\partial y_{2}} \frac{\partial}{\partial y_{3}} \prod_{i=1}^{N} \exp \left[\left(y_{i}+h\right) \sigma_{i}^{\prime}\right]\right]\right\} \\
= & K \cdot \bar{E}\left[\frac{\partial}{\partial x_{1}} \frac{\partial}{\partial y_{2}} \frac{\partial}{\partial y_{3}} g(\bar{x}, \bar{y}, \bar{z}, \bar{w})\right]
\end{aligned}
$$

where $K=2^{4 N} Z^{-4} \exp \left(-2 N J_{0}\right)$, and

$$
g(\bar{x}, \bar{y}, \bar{z}, \bar{w})=\prod_{i=1}^{N} \cosh \left(x_{i}+h\right) \cosh \left(y_{i}+h\right) \cosh \left(z_{i}+h\right) \cosh \left(w_{i}+h\right),
$$


and where we have used (3.2) twice for the $\bar{z}$ and $\bar{w}$ variables. Therefore, to prove (2.4), we take the same $K$ and $g$ and define

$$
\begin{aligned}
D\left(\frac{\partial}{\partial x_{l}}, \frac{\partial}{\partial y_{l}}, \frac{\partial}{\partial z_{l}}\right)= & \frac{\partial}{\partial x_{1}} \frac{\partial}{\partial x_{2}} \frac{\partial}{\partial x_{3}}-\frac{\partial}{\partial x_{1}} \frac{\partial}{\partial x_{2}} \frac{\partial}{\partial y_{3}}-\frac{\partial}{\partial x_{1}} \frac{\partial}{\partial y_{2}} \frac{\partial}{\partial y_{3}} \\
& -\frac{\partial}{\partial x_{1}} \frac{\partial}{\partial y_{2}} \frac{\partial}{\partial x_{3}}+2 \frac{\partial}{\partial x_{1}} \frac{\partial}{\partial y_{2}} \frac{\partial}{\partial z_{3}} .
\end{aligned}
$$

The representation of $u_{3}(1,2,3)$ with this particular $D$ is not unique, for $D$ may be replaced by any differential operator obtained by performing the same permutation to the three sets of variables $\left(x_{i}, y_{i}, z_{i}, w_{i}\right), i=1,2,3$. This follows from the permutation invariance of $g(\bar{x}, \bar{y}, \bar{z}, \bar{w})$ and of $f_{J}(\bar{x}) f_{J}(\bar{y}) f_{J}(\bar{z}) f_{J}(\bar{w})$.

For each $i=1, \ldots, N$, we define new variables $\left(\alpha_{i}, \beta_{i}, \gamma_{i}, \delta_{i}\right)$ by the formula

$$
\left(\begin{array}{l}
\alpha_{i} \\
\beta_{i} \\
\gamma_{i} \\
\delta_{i}
\end{array}\right)=\frac{1}{2}\left(\begin{array}{rrrr}
1 & 1 & 1 & 1 \\
-1 & 1 & -1 & 1 \\
-1 & -1 & 1 & 1 \\
1 & -1 & -1 & 1
\end{array}\right)\left(\begin{array}{l}
x_{i} \\
y_{i} \\
z_{i} \\
w_{i}
\end{array}\right)
$$

The orthogonal matrix in (3.6) is the direct product with itself of the matrix $2^{-\frac{1}{2}}\left(\begin{array}{rr}1 & 1 \\ -1 & 1\end{array}\right)$ which is analogous to the one used in [4]. In the Appendix we show that $2\left(\partial / \partial \beta_{1}\right)\left(\partial / \partial \gamma_{2}\right)\left(\partial / \partial \delta_{3}\right)$ corresponds to the differential operator $D$ given by (3.5). Also, $g(\bar{x}, \bar{y}, \bar{z}, \bar{w})$ goes over to

where

$$
\tilde{g}(\bar{\alpha}, \bar{\beta}, \bar{\gamma}, \bar{\delta})=\prod_{i=1}^{N} t\left(\alpha_{i}, \beta_{i}, \gamma_{i}, \delta_{i}\right),
$$

$$
\begin{aligned}
t\left(\alpha_{i}, \beta_{i}, \gamma_{i}, \delta_{i}\right)= & \cosh 2\left(\alpha_{i}+2 h\right)+\cosh 2 \beta_{i}+\cosh 2 \gamma_{i}+\cosh 2 \delta_{i} \\
& +\cosh \left[-\left(\alpha_{i}+2 h\right)+\beta_{i}+\gamma_{i}+\delta_{i}\right]+\cosh \left[\left(\alpha_{i}+2 h\right)-\beta_{i}+\gamma_{i}+\delta_{i}\right] \\
& +\cosh \left[\left(\alpha_{i}+2 h\right)+\beta_{i}-\gamma_{i}+\delta_{i}\right]+\cosh \left[\left(\alpha_{i}+2 h\right)+\beta_{i}+\gamma_{i}-\delta_{i}\right] .
\end{aligned}
$$

Since $f_{J}(\bar{x}) f_{J}(\bar{y}) f_{J}(\bar{z}) f_{J}(\bar{w})$ transforms to $f_{J}(\bar{\alpha}) f_{J}(\bar{\beta}) f_{J}(\bar{\gamma}) f_{J}(\bar{\delta}),(2.5)$ results. We claim that $t\left(\alpha_{i}, \beta_{i}, \gamma_{i}, \delta_{i}\right)$ is a sum of terms of the form

$$
A\left(\alpha_{i}+2 h\right)^{j_{\alpha}}\left(\beta_{i}\right)^{j_{\beta}}\left(\gamma_{i}\right)^{j_{\gamma}}\left(\delta_{i}\right)^{j_{\delta}}-B\left(\alpha_{i}+2 h\right)^{k_{\alpha}}\left(\beta_{i}\right)^{k_{\beta}}\left(\gamma_{i}\right)^{k_{\gamma}}\left(\delta_{i}\right)^{k_{\delta}}
$$

where $A$ and $B$ are non-negative coefficients, $j_{\alpha}, j_{\beta}, j_{\gamma}, j_{\delta}$ are non-negative even integers, and $k_{\alpha}, k_{\beta}, k_{\gamma}, k_{\delta}$ are positive odd integers. This is proved at the end of this section. Given this, the GHS inequality (1.4) follows. Indeed, $\tilde{g}$ is then a sum of terms of the form

$$
C \prod_{i=1}^{N}\left(\alpha_{i}+2 h\right)^{l_{i}}\left(\beta_{i}\right)^{m_{i}}\left(\gamma_{i}\right)^{n_{i}}\left(\delta_{i}\right)^{p_{\imath}}
$$

where $\Sigma l_{i}, \Sigma m_{i}, \Sigma n_{i}$, and $\Sigma p_{i}$ are either all odd or all even and the constant $C$ is either negative or positive, respectively. In the odd case, with $h \geqq 0$, the derivative operator $\left(\partial / \partial \beta_{1}\right)\left(\partial / \partial \gamma_{2}\right)\left(\partial / \partial \delta_{3}\right)$ working on (3.10) either gives zero or converts 
(3.10) into an expression containing an even number of $\beta_{i}{ }^{\prime} s, \gamma_{i}{ }^{\prime} s$, and $\delta_{i}{ }^{\prime} s$. Also, when the term $\Pi_{i}\left(\alpha_{i}+2 h\right)^{l_{l}}$ is expanded, there appear an even number of $\alpha_{i}^{\prime} s$ (as well as an odd number, which gives zero in (2.5)). Hence by (2.3) the contribution of (3.10) to the $\bar{E}$-expectation in (2.5) is non-positive. In the odd case with $h=0$ and in the even case with any $h \geqq 0$, the contribution of (3.10) to the $\bar{E}$-expectation in (2.5) is easily shown to be zero.

To prove Theorem 1, first assume $h>0$. In cases a) and b), regardless of the parity of $N_{1}, \tilde{D}$ operating on (3.10) either gives zero or converts (3.10) into an expression containing an even number of $\alpha_{i}{ }^{\prime}$ s, $\beta_{i}$ 's, $\gamma_{i}$ 's, and $\delta_{i}$ 's (again expand $\left.\Pi_{i}\left(\alpha_{i}+2 h\right)^{l_{i}}\right)$. Such a term gives a non-positive contribution in case a), a nonnegative contribution in case b), to the $\bar{E}$-expectation in (2.5). In case c), there are no non-zero contributions. When $h=0$, we must consider the parity of $N_{1}$ because then the $\alpha_{i}$ variables are on the same footing as the $\beta_{i}, \gamma_{i}$, and $\delta_{i}$ variables.

To prove (3.9), we expand the cosh terms in (3.8). To ease the notation, we drop the subscripts in $\alpha_{i}, \beta_{i}, \gamma_{i}$, and $\delta_{i}$. The first four terms in (3.8) give rise to terms of the form in (3.9), with $A>0$ and $B=0$. Each of the last four gives rise to terms of the form

where

$$
\begin{gathered}
A^{\prime}\left[\varepsilon_{1}{ }^{i}(\alpha+2 h)+\varepsilon_{2}{ }^{i} \beta+\varepsilon_{3}{ }^{i} \gamma+\varepsilon_{4}{ }^{i} \delta\right]^{2 n}, \\
A^{\prime} \geqq 0, n \geqq 0, i=1,2,3,4,
\end{gathered}
$$

But

$$
\varepsilon_{j}^{i}=\left\{\begin{array}{rr}
-1, & j=i, \\
1, & j \neq i .
\end{array}\right.
$$

$$
\begin{aligned}
\sum_{i=1}^{4}\left[\varepsilon_{1}{ }^{i}(\alpha+2 h)+\varepsilon_{2}{ }^{i} \beta+\varepsilon_{3}{ }^{i} \gamma+\varepsilon_{4}{ }^{i} \delta\right]^{2 n} & \\
= & B^{\prime} \sum \sum_{i=1}^{4}\left[\varepsilon_{1}{ }^{i}(\alpha+2 h)\right]^{m_{\alpha}}\left(\varepsilon_{2}{ }^{i} \beta\right)^{m_{\beta}}\left(\varepsilon_{3}{ }^{i} \gamma\right)^{m_{\gamma}}\left(\varepsilon_{4}{ }^{i} \delta\right)^{m_{\delta}},
\end{aligned}
$$

where $B^{\prime}>0$ and the outer summation extends over all non-negative integers $m_{\alpha}, m_{\beta}, m_{\gamma}, m_{\delta}$ with sum $2 n$. Hence, either none, two, or all four of the $m$ 's are odd. In the first and third cases, we have terms of the form in (3.9) $(A>0, B=0$, and $A=0, B>0$, respectively). If two of the $m$ 's are odd, then when the inner sum over $i$ is done, there results a zero. This completes the proof of the GHS inequality and of Theorem 1.

\section{Appendix}

We first do the calculation for the GHS inequalities, then for the GKS inequalities mentioned in the text. For convenience, rather than write partial derivatives like $\left(\partial / \partial \beta_{1}\right)\left(\partial / \partial \gamma_{2}\right)\left(\partial / \partial \delta_{3}\right)$ and $\left(\partial / \partial x_{1}\right)\left(\partial / \partial y_{2}\right)\left(\partial / \partial x_{3}\right)$, we write $\beta \gamma \delta$ and $x y x$, respectively.

GHS. The expression $\beta \gamma \delta$ goes over to

$$
\frac{1}{8}(-x+y-z+w)(-x-y+z+w)(x-y-z+w) \equiv T_{1}+T_{2}+T_{3},
$$

where the terms in $T_{1}$ contain only a single variable, those in $T_{2}$ two different variables, and those in $T_{3}$ three different variables. We see that

$$
T_{1}=\frac{1}{8}(x x x+y y y+z z z+w w w),
$$


which goes over to $x x x / 2$ after permutation. The terms in $T_{2}$ fall into three separate groups: those where the repeated variable appears in the first and second slot, in the first and third slot, and in the second and third slot, respectively. Taking into account the signs and permuting, we see that the three groups contribute $-x x y / 2,-x y x / 2$, and $-x y y / 2$, respectively. Finally, $T_{3}$ contributes $x y z$. Thus, $2 \beta \gamma \delta$ goes over to

$$
x x x-x x y-x y x-x y y+2 x y z
$$

which is $D$ in (3.5).

GKS. $\tilde{\mathscr{D}}=\partial / \partial \alpha_{1}$ goes over to $(x+y+z+w) / 2$, which is equivalent to $2 x$. Hence,

$$
c_{\mathscr{D}}=2\left\langle\sigma_{1}\right\rangle \text {. }
$$

$\tilde{\mathscr{D}}=\left(\partial / \partial \beta_{1}\right)\left(\partial / \partial \beta_{2}\right)$ goes over to $(-x+y-z+w)(-x+y-z+w) / 4$, which is equivalent to $x x-x y$. Hence

$$
c_{\mathscr{D}}=\left\langle\sigma_{1} \sigma_{2}\right\rangle-\left\langle\sigma_{1}\right\rangle\left\langle\sigma_{2}\right\rangle .
$$

Since we are in case b) of Theorem 1, the expressions in (A.4) and (A.5) are both non-negative.

\section{References}

1. Griffiths, R. B., Hurst, C.A., Sherman, S.: J. Math. Phys. 11, 790 (1970)

2. Preston, C. J.: Commun. math. Phys. 35, 253 (1974)

3. Lebowitz, J.L.: Commun. math. Phys. 35, 87 (1974)

4. Monroe, J.L., Siegert, A. J. F.: J. Stat. Phys. 10, 237 (1974)

5. Griffiths, R. B.: J. Math. Phys. 8, 478, 484 (1967). Kelly,D.G., Sherman, S.: J. Math. Phys. 9 , $466(1968)$

6. Monroe, J.L.: J. Math. Phys. 15, 998 (1974)

7. Fortuin, C. M., Kasteleyn,P.W., Ginibre, J.: Commun. math. Phys. 22, 89 (1971)

8. Cramér,H.: Mathematical methods in statistics, p. 118. Princeton: Princeton University Press, N.J. 1951

Communicated by G. Gallavotti

Richard S. Ellis

Department of Mathematics

Northwestern University

Evanston, Illinois 60201, USA
James L. Monroe

Department of Physics

Northwestern University

Evanston, Illinois 60201, USA

Note Added in Proof. One consequence of the GHS inequality is the negativity at $h=0$ of the Fourth Ursell Function $u_{4}(i, j, k, l)$. This also follows directly from Theorem 1. Indeed, $\tilde{\mathscr{D}}=\left(\partial / \partial \alpha_{i}\right)\left(\partial / \partial \beta_{j}\right)\left(\partial / \partial \gamma_{k}\right)\left(\partial / \partial \delta_{l}\right)$ can be shown to give rise to $u_{4}(i, j, k, l)$ at $h=0$. Since this $\tilde{\mathscr{D}}$ is covered by case a) of Theorem 1 , the negativity of $u_{4}$ follows. 